\title{
Random Growth and Random Matrices
}

\author{
Kurt Johansson
}

\begin{abstract}
We give a survey of some of the recent results on certain two-dimensional random growth models and their relation to random matrix theory, in particular to the Tracy-Widom distribution for the largest eigenvalue. The problems are related to that of finding the length of the longest increasing subsequence in a random permutation. We also give a new approach to certain results for the Schur measure introduced by Okounkov.
\end{abstract}

\section{Random Growth Models in the Plane}

\subsection{Eden-Richardson growth}

During the last twenty years there has been alot of interest in models where an object grow by some rule involving randomness. Basically, there are two types of models, non-local models like difusion-limited aggregation (DLA) and local models, which is our concern here. There are many types of local random growth models in the plane, see [23] for a review and more background. As an example consider the Eden-Richardson growth model, $[12,29]$, which is defined as follows. The shape $\Omega_{t}$ at time $t$ of the growing object is a connected set, which is the union of unit squares centered at points in $\mathbb{Z}^{2}$. Let $\partial \Omega_{t}$ denote the set of all unit squares, centered at integer points, which are adjacent to $\Omega_{t}$. In the continuous time version each square in $\partial \Omega_{t}$ is added to $\Omega_{t}$ independently of each other and with exponential waiting times; i.e. as soon as a square joins $\partial \Omega_{t}$ it's clock starts to tick and the square is added to $\Omega_{t}$ after a random time $T$ with the exponental distribution, $P[T>s]=e^{-s}$. In the discrete time version, at each time $t \in \mathbb{Z}^{+}$, each square in $\partial \Omega_{t-1}$, is added to $\Omega_{t-1}$ with probability $p=1-q$ independently of each other, and the resulting set is $\Omega_{t}$. At time $t=0$ we take $\Omega_{0}=[-1 / 2,1 / 2]^{2}$. In both cases the object grows linearly in time, [20], $\Omega_{t} / t \rightarrow A$, the asymptotic shape, as $t \rightarrow \infty$. We are interested in the roughness of $\Omega_{t}$, the fluctuations of $\Omega_{t}$ around $t A$. This growth model is equivalent with a certain first-passage site percolation model. With each site $(i, j) \in \mathbb{Z}^{2}$ we associate a random variable $\tau(i, j)$, which we think of as a random time. Variables associated with different sites are independent. A path $\pi$ from $(0,0)$ to $(M, N)$ is a sequence $\left\{p_{r}\right\}_{r=0}^{R} \subseteq \mathbb{Z}^{2}$ with $\left|p_{r}-p_{r-1}\right|=1$, $p_{0}=(0,0)$ and $p_{R}=(M, N)$. The first-passage time from $(0,0)$ to $(M, N)$ is

$$
T(M, N)=\min _{\pi} \sum_{p_{r} \in \pi} \tau\left(p_{r}\right) .
$$


If we take $\tau(0,0)=0$ and $P[\tau(i, j)>s)=e^{-s}$ (continuous time) or $\tau(i, j)=$ $w(i, j)+1$ with $P[w(i, j)=s]=(1-q) q^{s}, s \geq 0,0<q<1$ (discrete time), then

$$
\Omega_{t}=\left\{(M, N) \in \mathbb{Z}^{2} ; T(M, N) \leq t\right\}+\left[-\frac{1}{2}, \frac{1}{2}\right]^{2} .
$$

Thus, fluctuations in $\Omega_{t}$ can be translated into fluctuations of $T(M, N)$. It is comjectured that the standard deviation $S D(T(N, N)) \sim N^{\chi}$, as $N \rightarrow \infty$ with $\chi=1 / 3,[23,26]$. Since $T(N, N) \sim c N$ as $N \rightarrow \infty$, we see that the standard deviation grows like (mean $)^{1 / 3}$. This means that the longitudinal fluctuations of $\Omega_{t}$ are of order $t^{1 / 3}$, which is conjectured to be true generally for local two-dimensional random growth models. We can also consider the transversal fluctuations. Let $d_{N}$ be the maximal deviation of a all paths $\pi$, which are minimizers in (1) with $M=N \geq 0$, from the straight line $x=y$. It is conjectured that $d_{N}$ is of order $N^{\xi}$ with $\xi=2 / 3$, [24]. To prove that $\chi=1 / 3$ and $\xi=2 / 3$ in the Eden-Richardson growth model is an open problem. Below we will consider related models, where this, and more, can be rigorously proved.

\subsection{The corner growth model}

We can modify the model above by allowing growth only upwards or to the right. In (1) this corresponds to allowing only up/right paths $\pi$ in (1), i.e. $p_{r}-p_{r-1}=$ $(1,0)$ or $(0,1)$, which gives a so called directed first-passage percolation model. We can add one more restriction by allowing growth only in corners. In this model $\Omega_{0}=\mathbb{R}^{2} \backslash \mathbb{R}_{+}^{2}$ and we can add a square $Q=[m-1, m] \times[n-1, n]$ to $\Omega_{t}$ only if both $[m-2, m-1] \times[n-1, n] \subseteq \Omega_{t}$ and $[m-1, m] \times[n-2, n-1] \subseteq \Omega_{t}$, i.e. $Q$ lies in a "corner" of $\Omega_{t}$. For this model we have to replace (1) by

$$
G(M, N)=\max _{\pi} \sum_{(i, j) \in \pi} w(i, j),
$$

where the maximum is over all up/right paths from $(1,1)$ to $(M, N)$. Thus, we get instead what can be called a last-passage directed percolation model, and the random shape is given by

$$
\Omega_{t}=\left\{(M, N) \in \mathbb{Z}^{2} ; G(M, N)+M+N-1 \leq t\right\}+[-1,0]^{2},
$$

since all up/right paths from $(1,1)$ to $(M, N)$ contains the same number of points $M+N-1$. The continuous time case can be obtained by taking the limit $q \rightarrow 1$, see [16]. Note that if $q=1-1 / L$, then $L^{-1} w(i, j)$ converges, as $L \rightarrow \infty$ to an exponentially distributed random variable. As explained in [30], the corner growth model is equivalent with the discrete or continuous time totally asymmetric simple exclusion process (TASEP) and results for the growth model can be translated into results for the TASEP, [16].

In order to state the results we have to define the Tracy-Widom distribution. Let $\operatorname{Ai}(x)$ denote the Airy function and define the Airy kernel,

$$
A(x, y)=\int_{0}^{\infty} \operatorname{Ai}(x+t) \operatorname{Ai}(y+t) d t=\frac{\operatorname{Ai}(x) \operatorname{Ai}^{\prime}(y)-\operatorname{Ai}^{\prime}(x) \operatorname{Ai}(y)}{x-y} .
$$


The Tracy-Widom distribution is defined by the Fredholm determinant

$$
F(t)=\operatorname{det}(I-A)_{L^{2}(t, \infty)}, \quad t \in \mathbb{R} .
$$

Let $M$ be an $N \times N$ matrix from the Gaussian Unitary Ensemble (GUE), [25], where we put the measure $Z_{N}^{-1} \exp \left(-\operatorname{Tr} M^{2}\right) d M$ on the space of Hermitian matrices. The space of $N \times N$ Hermitian matrices is isomorphic to $R^{N^{2}}$ and $d M$ is Lebesgue measure on this space. If $\lambda_{\max }$ is the largest eigenvalue of $M$, then

$$
\lim _{N \rightarrow \infty} P\left[\sqrt{2} N^{1 / 6}\left(\lambda_{\max }-\sqrt{2 N}\right) \leq t\right]=F(t),
$$

see $[35]$.

We can now state the main theorem for the corner growth model.

Theorem 1.1. [16]. For each $q \in(0,1), \gamma \geq 1$ and $s \in \mathbb{R}$,

$$
\lim _{N \rightarrow \infty} P\left[\frac{G([\gamma N], N)-N \omega(\gamma, q)}{\sigma(\gamma, q) N^{1 / 3}} \leq s\right]=F(s),
$$

where

and

$$
\omega(\gamma, q)=\frac{(1+\sqrt{q \gamma})^{2}}{1-q}-1
$$

$$
\sigma(\gamma, q)=\frac{q^{1 / 6} \gamma^{-1 / 6}}{1-q}(\sqrt{\gamma}+\sqrt{q})^{2 / 3}(1+\sqrt{q \gamma})^{2 / 3}
$$

From $\omega(\gamma, q)$ we can compute the asymptotic shape $A$ for the corner growth model, which was also done in [32], and we obtain $A \cap \mathbb{R}_{+}^{2}=\left\{(x, y) \in \mathbb{R}_{+}^{2} ; y+\right.$ $2 \sqrt{q x y}+x \leq 1-q\}$. Note that in this model the standard deviation goes like $\sim(\text { mean })^{1 / 3}$, so we have a proof of $\chi=1 / 3$. Also we see from $(8)$ that $G(M, N)$, for $M$ and $N$ large, behaves like the largest eigenvalue of a big random hermitian matrix. There are analogous results for the continuous time case and the TASEP, see $[16]$.

Theorem 1.1 is proved using the following representation of the distribution function for $G(M, N), M \geq N$,

$$
P[G(M, N) \leq n]=\frac{1}{Z_{M, N}} \sum_{h \in\{0, \ldots, n+N-1\}^{N}} \Delta_{N}(h)^{2} \prod_{j=1}^{N}\left(\begin{array}{c}
h_{j}+M-N \\
h_{j}
\end{array}\right) q^{h_{j}},
$$

where $\Delta_{N}(h)=\prod_{1 \leq i<j \leq N}\left(h_{i}-h_{j}\right)$ is the Vandermonde determinant and $Z_{M, N}$ is a normalization constant. How this formula is obtained is described in the next section. The formula (11) should be compared with the formula for the distribution function for the largest eigenvalue of a GUE-matrix,

$$
P\left[\lambda_{\max } \leq t\right]=\frac{1}{Z_{N}} \int_{(-\infty, t]^{N}} \Delta_{N}(x)^{2} \prod_{j=1}^{N} e^{-x_{j}^{2}} d^{N} x .
$$

Note that in the continuous time case we obtain, by taking a limit $q \rightarrow 1$ in (11), a similar integral giving the largest eigenvalue in the Laguerre ensemble, [16]. 
The right hand side of (12) can also be written as

$$
\frac{1}{Z_{N}} \int_{(-\infty, t]^{N}} \exp \left(\sum_{j \neq k} \log \left|x_{j}-x_{k}\right|^{-1}+\sum_{j} x_{j}^{2}\right) d^{N} x,
$$

which leads to Dyson's Coulomb gas interpretation. We see that $\lambda_{\max }$ is the position of the rightmost charge in a logarithmic Coulomb gas on the line confined by a quadratic external potential. In (11) we get instead a discrete Coulomb gas on $\mathbb{N}$, also confined by an external potential. At the edge of the support of the charges the density is low, so we expect the discrete Coulomb gas to be well approximated by a continuous Coulomb gas in this region, and thus, heuristically, $G(M, N)$ and $\lambda_{\max }$ should behave similarly.

To establish this rigorously one can proceed as follows. A standard computation in random matrix theory, [25, ch. 5], shows that the right hand side of (12) equals a certain Fredholm determinant and this is used to prove (7). Carrying out the same computation for (11), [16], gives

$$
P[G(M, N) \leq n]=\operatorname{det}\left(I-K_{M, N}\right)_{\ell(\{n+N, n+N+1, \ldots\})},
$$

where $K_{M, N}$ is the operator on $\ell^{2}(\mathbb{N})$ with kernel

$$
\begin{aligned}
K_{M, N}(x, y) & =\frac{\kappa_{N-1}}{\kappa_{N}} \frac{M_{N}(x) M_{N-1}(y)-M_{N-1}(x) M_{N}(y)}{x-y} \\
& \times\left[\left(\begin{array}{c}
x+M-N \\
x
\end{array}\right)\left(\begin{array}{c}
y+M-N \\
y
\end{array}\right) q^{x+y}\right]^{1 / 2},
\end{aligned}
$$

for $x, y \in \mathbb{N}$. Here $M_{n}(x)=\kappa_{n} x^{n}+\ldots$ are the normalized, discrete orthogonal polynomials with respect to the weight $\left(\begin{array}{c}x+M-N \\ x\end{array}\right) q^{x}$ on $\mathbb{N}$. They are multiples of the standard Meixner polynomials, [10]. Theorem 1.1 is proved by analyzing the asymptotics of the Fredholm determinant (14), using he fact that the asymptotics of the Meixner kernel, (15) can be analyzed using the integral formula for the Meixner poynomials, [16].

\subsection{Hammersley's model}

Consider a Poisson process with intensity $\alpha$ in the unit square $[0,1]^{2}$. An up/right path from $(0,0)$ to $(1,1)$ through the points is a sequence $\left\{\left(x_{k}, y_{k}\right)\right\}_{k=1}^{L}$ of Poisson points such that $x_{k} \leq x_{k+1}$ and $y_{k} \leq y_{k+1}$ for each $k$. Let $L(\alpha)$ denote the maximum number of points in such a path. This random variable was introduced in [15] to study random permutations. In fact, if we condition the number of points in the square to be $N$, then $L(\alpha)$ has the same distribution as the length of the longest increasing subsequence in a random permutation from $S_{N}$ with uniform distribution, see also [1]. Hammersley's model can also be obtained as a limit of the corner growth model by taking $q=\alpha / N^{2}$ and letting $N$ go to infinity. With this choice of $q$ the $N \times N$ matrix $(w(i, j))_{i, j=1}^{N}$ has, with probability going to 1 as $N \rightarrow \infty$, at most one 1 in each row and column, and all other elements are 0 . From this it is not hard to see that $G(N, N)$ converges to $L(\alpha)$. Thus one approach to 
the results for $L(\alpha)$ is to take $M=N$ and $q=\alpha / N^{2}$ in (14) and then compute the limit as $N \rightarrow \infty$, see [18] and [19] for a review.

The distribution function for $L(\alpha)$ also has another representation, [14], as a Toeplitz determinant,

$$
P[L(\alpha) \leq n]=D_{n}\left(e^{2 \sqrt{\alpha} \cos \theta}\right) \doteq \operatorname{det}\left(\frac{1}{2 \pi} \int_{-\pi}^{\pi} e^{2 \sqrt{\alpha} \cos \theta-i(j-k) \theta} d \theta\right)_{j, k=1}^{N}
$$

The asymptotics of the Toeplitz determinant in (16) can be analyzed using the steepest descent method for Riemann-Hilbert problems, [11], and this leads to a proof of

Theorem 1.2. [5]. For all $t \in \mathbb{R}$,

$$
\lim _{\alpha \rightarrow \infty} P\left[L(\alpha) \leq 2 \sqrt{\alpha}+t \alpha^{1 / 6}\right]=F(t),
$$

where $F(t)$ is the Tracy-Widom distribution (6), and also all moments of $L(\alpha)$ converge.

Note that the standard deviation of $L(\alpha)$ is $\sim c \alpha^{1 / 6}=c(\sqrt{\alpha})^{1 / 3}$ and the mean is $\sim 2 \sqrt{\alpha}$, so we have the same exponent $\chi=1 / 3$ as above. This theorem was the first case where this exponent was rigorously verified. In the proof of (17) in [5] another expression for the Tracy-Widom distribution is obtained, namely

$$
F(t)=\exp \left(-\int_{t}^{\infty}(x-t) u(x)^{2} d x\right)
$$

where $u(x)$ is the solution of the Painlevé II equation $u^{\prime \prime}=x u+2 u^{3}$, which satsifies $u(x) \sim \operatorname{Ai}(x)$ as $x \rightarrow \infty$. The fact that (6) and (18) are equal is proved in [35]. It is also possible to consider random permutations (or the Hammersley model) with symmetry restrictions and restrictions on the number of fixed points, $[3,4]$. For these models one can also obtain the GOE and GSE largest eigenvalue distributions, [35], as limits besides the GUE distribution discussed above, see [4].

Hammersley's model has an interesting interpretation as a two-dimensional growth model called polynuclear growth (PNG), see [28]. Some of the symmetrized models mentioned above also have an interpretation in this context, [28]. In Hammersley's model we can also obtain the exponent $\xi$ discussed above, by looking at the largest deviation of any maximal path from the diagonal. Using estimates from [5] and ideas from $[24,37]$ it is possible to prove that $\xi=2 / 3$ in this model, see $[17]$.

The rigorous results discussed above are of course proved for very special models. On the other hand one would expect the results to hold more generally, [22], [16, Conjecture 1.9], [28], since limiting laws should have some degree of universality. To understand this universality rigorously is an interesting open problem. 


\section{Analysis of the Corner Growth Model}

Let $\lambda=\left(\lambda_{1}, \ldots, \lambda_{n}\right)$ be a partition of $k$, i.e. $\lambda_{1} \geq \lambda_{2} \geq \cdots \geq \lambda_{n} \geq 0, \lambda_{j} \in \mathbb{N}$ and $\sum_{j} \lambda_{j}=k$. The Young or Ferrers diagram corresponding to $\lambda$ is the set $\cup_{j=1}^{n}\left\{(i, j) ; 1 \leq j \leq \lambda_{n+1-j}\right\}$, which we also denote by $\lambda$. A semistandard Young tableaux $T$ of shape $\lambda, \operatorname{sh}(T)=\lambda$, with elements in $\{1, \ldots, N\}$ is a map $T: \lambda \rightarrow$ $\{1, \ldots, N\}$ such that $T(i, j) \leq T(i+1, j)$ and $T(i, j)>T(i, j+1)$. Let $m_{r}(T)$ denote the number of points $(i, j) \in \lambda$, such that $T(i, j)=r$, see $[13,31]$ or $[34]$ for more details. The Robinson-Schensted-Knuth (RSK) corresponence, [21], sets up a bijection between integer $M \times N$ matrices $W=(w(i, j))$ and pairs $(T, S)$ of semistandard Young tableaux of the same shape $\lambda$, where $T$ has elements in $\{1, \ldots, N\}$ and $S$ has elements in $\{1, \ldots, M\}$. This bijection has the property that $G(M, N)$ defined by (3) is equal to $\lambda_{1}$, see [16], and furthermore $\sum_{j} w(i, j)=$ $m_{i}(S), \sum_{i} w(i, j)=m_{j}(T)$.

Consider a generalization of the corner growth model defined above, where $w(i, j)$ is geometrically distributed with parameter $x_{i} y_{j}$, i.e. $P[w(i, j)=s]=$ $\left(1-x_{i} y_{j}\right)\left(x_{i} y_{j}\right)^{s}, s \in \mathbb{N}$. Here $x_{i}, y_{i}, i \geq 1$, are given numbers in $(0,1)$. From the facts above we obtain

$$
\begin{aligned}
& P[G(N, N) \leq n]=\sum_{W ; G(N, N) \leq n} P[W] \\
& =\prod_{i, j=1}^{N}\left(1-x_{i} y_{j}\right) \sum_{W ; G(N, N) \leq n} \prod_{i=1}^{N} x_{i}^{\sum_{j} w(i, j)} \prod_{j=1}^{N} y_{j}^{\sum_{i} w(i, j)} \\
& =\prod_{i, j=1}^{N}\left(1-x_{i} y_{j}\right) \sum_{\lambda ; \lambda_{1} \leq n}\left(\sum_{S ; \operatorname{sh}(S)=\lambda} \prod_{i=1}^{N} x_{i}^{m_{i}(S)}\right)\left(\sum_{T ; \operatorname{sh}(T)=\lambda} \prod_{j=1}^{N} y_{j}^{m_{j}(T)}\right)
\end{aligned}
$$

In (2.1) we can recognize one possible definition of the Schur polynomial. Given $\lambda=\left(\lambda_{1}, \ldots, \lambda_{N}\right)$, a partition of $k$, the Schur polynomial $s_{\lambda}\left(x_{1}, \ldots, x_{N}\right)$ is a homogeneous symmetric polynomial of degree $k$, defined by

$$
s_{\lambda}\left(x_{1}, \ldots, x_{N}\right)=\sum_{S ; \operatorname{sh}(S)=\lambda} \prod_{i=1}^{N} x_{i}^{m_{i}(S)}=\frac{1}{\Delta_{N}(x)} \operatorname{det}\left(x_{i}^{\lambda_{j}+N-j}\right)_{i, j=1}^{N} .
$$

The second equality is the Jacobi-Trudi identity, [31, 34]. Combining (19) and (20), we obtain

$$
P[G(N, N) \leq n]=\prod_{i, j=1}^{N}\left(1-x_{i} y_{j}\right) \sum_{\lambda ; \lambda_{1} \leq n} s_{\lambda}(x) s_{\lambda}(y),
$$

$[16,3]$. In [3] many variations of this formula are given. Note that we can think of

$$
P_{S}[\lambda]=\prod_{i, j=1}^{N}\left(1-x_{i} y_{j}\right) s_{\lambda}(x) s_{\lambda}(y)
$$


as a probability measure on all partitions with at most $N$ non-zero parts. This is the Schur measure introduced in [27], where it is defined on the set of all partitions and $x_{i}, y_{i}$ are allowed to be complex. As discussed in [7], the Schur measure generalizes many other measures on partitions motivated by representation theory, see [6], including the classical Plancherel measure, which corresponds to random permutations, [8]. The Poissonized Plancherel measure can be obtained as a limit of (22) with $x_{i}=y_{i}=\sqrt{\alpha} / N$, see $[18,19]$.

Note that if we put $y_{i}=0, M<i \leq N, y_{i}=\sqrt{q}, 1 \leq i \leq M$ and $x_{i}=\sqrt{q}$, $1 \leq j \leq N$ in (22) we get the distribution function for $G(N, M)$ of section 1 . Using the second equality in (20) we can rewrite the right hand side of (22) and obtain (11), [16]. The crucial fact in going from (11) to (14) is the fact that the measure in (11) has determinantal correlation functions, [25], see [33] for review on random point fields with determinantal correlation functions. In fact, this is true also for the Schur measure (22) as proved in [27]. In the next section we will give a new proof of this, and actually we will see that it is possible to go directly from (21) to a Fredholm determinant.

\section{The Schur Measure}

Our analysis of the Schur measure is based on [36], which is an alternative to the approach in [25]. We start by outlining some results in [36]. Let $\mu$ be a measure on the space $\Omega$ and let $f, \phi_{j}, \psi_{j}, j \geq 1$ be integrable functions from $\Omega$ to $\mathbb{C}$. The following identity, [2], is central.

$$
\begin{aligned}
Z_{N}[f] & \doteq \frac{1}{N !} \int_{\Omega^{N}} \operatorname{det}\left(\phi_{j}\left(t_{k}\right)\right)_{j, k=1}^{N} \operatorname{det}\left(\psi_{j}\left(t_{k}\right)\right)_{j, k=1}^{N} \prod_{j=1}^{N} f\left(t_{j}\right) d \mu\left(t_{j}\right) \\
& =\operatorname{det}\left(\int_{\Omega} \phi_{j}(t) \psi_{k}(t) f(t) d \mu(t)\right)_{j, k=1}^{N} .
\end{aligned}
$$

Let

$$
A=\left(\int_{\Omega} \phi_{j}(t) \psi_{k}(t) d \mu(t)\right)_{j, k=1}^{N}
$$

and assume that $A$ is invertible with inverse $A^{-1}=\left(\mu_{j k}\right)_{j, k=1}^{N}$. Set

$$
K_{N}(u, v)=\sum_{j, k=1}^{N} \psi_{k}(u) \mu_{k j} \phi_{j}(v) .
$$

The arguments in [36] show that, writing $f=1+g$,

$$
\frac{Z_{N}[1+g]}{Z_{N}[1]}=\operatorname{det}\left(I+K_{N} g\right)_{L^{2}(\Omega, \mu)},
$$

where the right hand side is a Fredholm determinant on $L^{2}(\Omega, \mu)$ and $K_{N} g$ is the operator which is first multiplication by $g$ and the application of the operator with 
kernel $K_{N}(u, v)$. Think of

$$
\rho_{N}\left(t_{1}, \ldots, t_{N}\right) d \mu^{N}(t)=\frac{1}{N ! Z_{N}[1]} \operatorname{det}\left(\phi_{j}\left(t_{k}\right)\right)_{j, k=1}^{N} \operatorname{det}\left(\psi_{j}\left(t_{k}\right)\right)_{j, k=1}^{N} d \mu^{N}(t)
$$

as a complex measure on $\Omega^{N}$. It follows from (26), [36], that $\rho_{N} d \mu^{N}$ has marginal distributions given by

$$
\begin{aligned}
\rho_{k}\left(t_{1}, \ldots, t_{k}\right) d \mu^{k}(t) & \doteq \int_{\Omega^{N-k}} \rho_{N}(t) d \mu\left(t_{k+1} \ldots d \mu\left(t_{N}\right)\right. \\
& =\frac{(N-k) !}{N !} \operatorname{det}\left(K_{N}\left(t_{i}, t_{j}\right)\right)_{i, j=1}^{k} d \mu^{k}(t) .
\end{aligned}
$$

Let $A^{(j k)}$ be the matrix obtained by deleting row $j$ and column $k$ in the matrix $A$. Then, by (25) and the standard formula for the inverse of a matrix,

$$
K_{N}(u, v)=\sum_{j, k=1}^{N}(-1)^{j+k} \psi_{k}(u) \frac{\operatorname{det} A^{(j k)}}{\operatorname{det} A} \phi_{j}(v) .
$$

If we take $\phi_{j}(v)=y_{j}^{v}, \psi_{k}(u)=x_{k}^{u}$ and $d \mu$ to be the counting measure on $\mathbb{N}$, then (27) becomes the Schur measure. To see this use the second equality in (20), the Jacobi-Trudi identity, set $t_{j}=\lambda_{j}+N-j$, and note that the Schur measure is a symmetric function of the $t_{i}$ :s. Hence we can regard the Schur measure, (22) as a measure on $\mathbb{N}^{N}$, which we denote by $\rho_{N}^{\mathrm{Sc}}\left(t_{1}, \ldots, t_{N}\right), t \in \mathbb{N}^{N}$. Note that with this choice,

$$
\begin{aligned}
Z_{N}[1] & =\operatorname{det} A=\operatorname{det}\left(\sum_{t=0}^{\infty} y_{j}^{t} x_{k}^{t}\right)=\operatorname{det}\left(\frac{1}{1-y_{j} x_{k}}\right) \\
& =\frac{\Delta_{N}(x) \Delta_{N}(y)}{\prod_{j, k=1}^{N}\left(1-x_{j} y_{k}\right)},
\end{aligned}
$$

by the formula for a Cauchy determinant, so we really get (22) with the right normalization. We see that $\operatorname{det} A^{(j k)}$ is also a Cauchy determinant and (29) becomes, after cancellation of common factors,

$$
K_{N}(u, v)=\sum_{r, s=1}^{N} y_{s}^{v} x_{r}^{u} \frac{\prod_{j}\left(1-x_{j} y_{s}\right)\left(1-x_{r} y_{j}\right)}{\left(1-x_{r} y_{s}\right) \prod_{j \neq r}\left(x_{r}-x_{j}\right) \prod_{j \neq s}\left(y_{s}-y_{j}\right)} .
$$

We can rewrite this using the residue theorem. Let $\gamma_{2}$ be the circle $|w|=1$ and $\gamma_{1}^{\prime}$ the circle $|\zeta|=(1+\epsilon)^{-1}$, where $\epsilon>0$ is chosen so that $\left|y_{j}\right|<(1+\epsilon)^{-1}$ for $1 \leq j \leq N$. Both curves have positive orientation. An application of the residue theorem shows that the right hand side of (31) is

$$
K_{N}(u, v)=\frac{1}{(2 \pi i)^{2}} \int_{\gamma_{1}^{\prime}} d \zeta \int_{\gamma_{2}} d w \frac{w^{u} \zeta^{v}}{1-\zeta w} \prod_{j=1}^{N}\left(\frac{1-w y_{j}}{w-x_{j}}\right)\left(\frac{1-x_{j} \zeta}{\zeta-y_{j}}\right) .
$$


If $\gamma_{1}$ is the circle $|z|=1+\epsilon$ and we put $z=1 / \zeta$, we get from (32)

$$
K_{N}(u, v)=\frac{1}{(2 \pi i)^{2}} \int_{\gamma_{1}} d \zeta \int_{\gamma_{2}} d w \frac{w^{u}}{z^{v+1}} \frac{1}{z-w} \prod_{j=1}^{N}\left(\frac{1-w y_{j}}{w-x_{j}}\right)\left(\frac{z-x_{j}}{1-y_{j} z}\right)
$$

and we have rederived the result in [27].

By (21), the definition of the Schur measure and the remarks above, we obtain

$$
P[G(N, N) \leq n]=\sum_{t \in \mathbb{N}^{N}} P_{N}^{\mathrm{Sc}}\left(t_{1}, \ldots, t_{N}\right) \prod_{j=1}^{N}\left(1-\chi_{[n+N, \infty)}\left(t_{j}\right)\right) .
$$

Hence, by (26),

$$
P[G(N, N) \leq n]=\operatorname{det}\left(I-K_{N}\right) \ell(\{n+N, n+N+1, \ldots\})
$$

with $K_{N}$ given by (33). If we put $y_{i}=0, M<i \leq N, y_{i}=\sqrt{q}, 1 \leq i \leq M$ and $x_{i}=\sqrt{q}, 1 \leq j \leq N$, this formula can be used to prove theorem 1.1. The asymptotics can be analyzed by a saddle-point argument of the integral in the rght hand side of (33). Note that in this approach we do not need to know anything about Meixner polynomials. After some manipulation, where we use the integral formula for Meixner polynomials, it is possible to obtain the representation (15) for the kernel, see [7].

Let $\lambda^{\prime}$ denote the conjugate partition to $\lambda$, i.e. $\lambda_{j}^{\prime}=$ the length of the $j$ :th column in $\lambda$, and let $\ell(\lambda)$ denote the number of non-zero-parts in $\lambda$. We have

$$
\sum_{\lambda ; \lambda_{1} \leq n} s_{\lambda}(x) s_{\lambda}(y)=\sum_{\mu ; \ell(\mu) \leq n} s_{\mu^{\prime}}(x) s_{\mu^{\prime}}(y) .
$$

Let $e_{k}(x)$ denote the $k$ :th elementary symmetric function, i.e.

$$
\prod_{j=1}^{N}\left(1+z x_{j}\right)=\sum_{k=0}^{N} e_{k}(x) z^{k}
$$

and $e_{k}(x)=0$ if $k<0, k>N$. Then, there is a third formula for the Schur polynomial, [31, 34],

$$
s_{\mu^{\prime}}(x)=\operatorname{det}\left(e_{t_{i}-j}(x)\right)_{i, j=1}^{N},
$$

where $\mu=\left(\mu_{1}, \ldots, \mu_{N}\right)$ and $t_{i}=\mu_{i}+N-i$. As described in [3], we can now use formula (23) to derive Gessel's formula. Inserting (38) into (36) and using (23) gives

$$
\sum_{\lambda ; \lambda_{1} \leq n} s_{\lambda}(x) s_{\lambda}(y)=\operatorname{det}\left(\sum_{m=0}^{\infty} e_{m-j}(x) e_{m-k}(y)\right)_{j, k=1}^{n} .
$$

Using (21) we find,

$$
P[G(N, N) \leq n]=\prod_{j, l=1}^{N}\left(1-x_{j} y_{k}\right) \operatorname{det}\left(\sum_{m=0}^{\infty} e_{m}(x) e_{m+j-k}(y)\right)_{j, k=1}^{n} .
$$


Note that the determinant in the right hand side of (40) is a Toeplitz determinant. If we set

$$
f(z)=\prod_{j=1}^{N}\left(1+x_{j} / z\right)\left(1+y_{j} z\right)
$$

then, by (37) and (40),

$$
P[G(N, N) \leq n]=\prod_{j, l=1}^{N}\left(1-x_{j} y_{k}\right) D_{n}\left(f\left(e^{i \theta}\right)\right) .
$$

If we take $x_{j}=y_{j}=\sqrt{\alpha} / N$ and let $N \rightarrow \infty$ we obtain (16). Combining (35) and (42) we find,

$$
D_{n}\left(f\left(e^{i \theta}\right)\right)=\prod_{j, l=1}^{N} \frac{1}{\left(1-x_{j} y_{k}\right)} \operatorname{det}\left(I-K_{N}\right)_{\ell^{2}(\{n+N, n+N+1, \ldots\})} .
$$

This is the Borodin-Okounkov identity, which can be extended to more general $f$ by taking appropriate limits, [7], see also [9] for a completely different derivation.

\section{References}

[1] D. Aldous, P. Diaconis, Longest increasing subsequences: From patience sorting to the Baik-Deift-Johansson theorem, Bull. AMS, 36 (1999), 199-213.

[2] C. Andréief, Note sur une relation les intégrales définies des produits des fonctions, Mém. de la Soc. Sci. Bordeaux, 2 (1883), 1-14.

[3] J. Baik, E. Rains, Algebraic aspects of increasing subsequences, math.CO/9905083.

[4] J. Baik, E. Rains, The asymptotics of monotone subsequences of involutions, math.CO/9905084.

[5] J. Baik, P. A. Deift, K. Johansson, On the distribution of the length of the longest increasing subsequence in a random permutation, J. Amer. Math. Soc., 12, (1999), 1119-1178.

[6] A. Borodin, G. Olshanski, Z-measures on partitions, Robinson-Schensted-Knuth correspondence, and $\beta=2$ random matrix ensembles, math.CO/9905189.

[7] A. Borodin, A. Okounkov, A Fredholm determinant formula for Toeplitz determinants, math.CA/9907165.

[8] A. Borodin, A. Okounkov, G. Olshanski, On asymptotics of Plancherel measures for symmetric groups, math.CO/990532.

[9] E. L. Basor, H. Widom, On a Toeplitz determinant identity of Borodin and Okounkov, preprint, math.FA/9909010.

[10] T. S. Chihara, An Introduction to Orthogonal Polynomials, New York, gordon and breach, 1978.

[11] P. A. Deift, X. Zhou, A Steepest descent method for oscillatory Riemann-Hilbert problems, Ann. Math., 137, (1993), 295-368. 
[12] M. Eden, in Proceedings of the Fourth Berkeley Symposium on Mathematical Statistics and Probability, Ed. F. Neyman, vol. iV, university of California Press, Berkeley, 1961.

[13] W. Fulton, Young Tableaux, London Mathematical Society, Student Texts 35, Cambridge Univ. Press, 1997.

[14] I. M. Gessel, Symmetric functions and P-recursiveness, J. Combin. Theory Ser. A, 53, (1990), 257-285.

[15] J. M. Hammersley, A few seedlings of research, In Proc. Sixth Berkeley Symp. Math. Statist. and Probability, , Volume 1, pp. 345-394, University of California Press, 1972.

[16] K. Johansson, Shape fluctuations and random matrices, Commun. Math. Phys., 209, (2000), 437-476.

[17] K. Johansson, Transversal fluctuations for increasing subsequences on the plane, math.PR/9910146, to appear in Probab. Th. and Rel. Fields.

[18] K. Johansson, Discrete orthogonal polynomial ensembles and the Plancherel measure, preprint, math.CO/9906120, to appear in Annals of Math.

[19] K. Johansson, Random permutations and the discrete Bessel kernel, to appear in the MSRI volume on random matrices, Cambridge University Press.

[20] H. Kesten, Aspects of first passage percolation, in Lecture Notes in Mathematics vol 1180, Springer-Verlag, Berlin, 1986.

[21] D. E. Knuth, Permutations, Matrices and Generalized Young Tableaux, Pacific J. Math., 34, (1970), 709-727.

[22] J. Krug, P. Meakin, T. Halpin-Healy, Amplitude universality for driven interfaces and directed polymers in random media, Phys. Rev. A, 45 (1992), 638-653.

[23] J. Krug, H. Spohn, Kinetic Roughening of Growing Interfaces. In: Solids far from Equilibrium: Growth, Morphology and Defects, Ed. C Godrèche, Cambridge, cambridge University Press, 1992, 479-582.

[24] C. Licea, C. M. Newman, M. S. T. Piza, Superdiffusivity in first-passage percolation, Probab. Th. and Rel. Fields, 106 (1996), 559-591.

[25] M. L. Mehta, Random Matrices, 2nd ed., Academic Press, San Diego 1991.

[26] C. M. Newman, M. S. T. Piza, Divergence of shape fluctuations in two dimensions, Ann. Prob., 23 (1995), 977-1005.

[27] A. Okounkov, Infinite wedge and measures on partitions, math.RT/9907127.

[28] M. Prähofer, H. Spohn, Universal distributions for growth processes in $1+1$ dimensions and random matrices, preprint 1999, cond-mat/9912264.

[29] D. Richardson, Random growth in a tesselation, Proc. Camb. Phil. Soc., 74 (1973), 515-528.

[30] H. Rost, Non-Equilibrium Behaviour of a Many Particle Process: Density Profile and Local Equilibria, Zeitschrift f. Wahrsch. Verw. Geb., 58 (1981), 41-53.

[31] B. Sagan, The Symmetric Group, Brooks/Cole Publ. Comp., 1991.

[32] T. Seppäläinen, Coupling the totally asymmetric simple exclusion process with a moving interface, Markov Process. Rel. Fields, 4 (1998), 592-628.

[33] A. Soshnikov, Determinantal random point fields, math.PR/0002099.

[34] R. P. Stanley, Enumerative Combinatorics, Vol. 2, Cambridge University Press, 1999. 
[35] C. A. Tracy, H. Widom, Level Spacing Distributions and the Airy Kernel, Commun. Math. Phys., 159, (1994), 151-174.

[36] C. A. Tracy, H. Widom, Correlation Functions, Cluster Functions, and Spacing Distributions for Random Matrices, J. Statist. Phys., 92, (1998), 809-835.

[37] M. V. Wüthrich, Scaling identity for crossing Brownian motion in a Poissonian potential, Probab. Th. and Rel. Fields, 112 (1998), 299-319.

Department of Mathematics,

Royal Institute of Technology,

S-100 44 Stockholm, Sweden

E-mail address: kurtj@math.kth.se 